\title{
Overexpression of Napsin A resensitizes drug-resistant lung cancer A549 cells to gefitinib by inhibiting EMT
}

\author{
LINSHUI ZHOU* ${ }^{*}$ XIN LV* ${ }^{*}$ JUNCHAO YANG, YUANHONG ZHU, ZHEN WANG and TINGZHEN XU \\ Department of Respiratory Medicine, The First Affiliated Hospital of Zhejiang Chinese Medicine University, \\ Hangzhou, Zhejiang 310006, P.R. China
}

Received April 16, 2017; Accepted March 7, 2018

DOI: $10.3892 / \mathrm{ol} .2018 .8963$

\begin{abstract}
Lung cancer is one of the most common malignant tumors and also the leading cause of cancer-related deaths in the world. Epidermal growth factor receptor tyrosine kinase inhibitors (EGFR-TKI), such as gefitinib, have been used in the therapy of lung cancer. However, the acquisition of drug resistance is a major limitation in the clinical efficiency of EGFR-TKIs. Epithelial-mesenchymal transition (EMT) has been demonstrated to be an underlying mechanism of acquired resistance. A previous study has reported that Napsin A expression can inhibit EMT in lung cancer cells. The present study therefore investigated the effect of Napsin A on the sensitivity of EGFR-TKI-resistant lung cancer cells. First, a drug-resistant lung cancer cell line was generated using the EGFR-TKI gefitinib on A549 cells (termed here A549-GFT). EMT was demonstrated to be induced in the drug resistant A549-GFT cells, evidenced by reduced E-cadherin expression and increased Vimentin expression compared with control A549 cells. Next, Napsin A was overexpressed in the cells by transfection of the Napsin A-expression vector, PLJM1-Napsin A. Western blot analysis confirmed that the protein expression levels of Napsin A were significantly elevated in the Napsin A-overexpressing cells. Cell proliferation and apoptosis assays were performed to evaluate the effect of Napsin A overexpression on resistant A549 cells. The results of MTT assay demonstrated that Napsin A overexpression inhibited the proliferation of A549 and drug-resistant A549-GFT cells and that the proliferation of Napsin A-overexpressing A549-GFT cells was significantly inhibited by gefitinib treatment compared with control
\end{abstract}

Correspondence to: Dr Zhen Wang, Department of Respiratory Medicine, The First Affiliated Hospital of Zhejiang Chinese Medicine University, 54 Youdian Road, Hangzhou, Zhejiang 310006, P.R. China

E-mail: wangzhenz77@163.com

${ }^{*}$ Contributed equally

Key words: Napsin A, lung cancer, drug resistant, gefitinib, epithelial-mesenchymal transition
A549-GFT cells. The results from the Annexin V/propidium iodide double staining apoptosis assay indicated that Napsin A overexpression enhanced gefitinib-induced apoptosis in A549-GFT cells. Additionally, EMT was reversed following Napsin A expression in A549-GFT cells, as evidenced by the restoration of E-cadherin and downregulation of Vimentin expression. Further investigation demonstrated that Napsin A overexpression resulted in inhibition of focal adhesion kinase, a critical factor in integrin signaling, in the resistant A549-GFT cells. These data suggested that Napsin A resensitized the drug-resistant A549-GFT cells to gefitinib, possibly by reversing EMT via integrin signaling inhibition. Therefore, Napsin A combined with a TKI may be a more effective treatment strategy for lung cancer.

\section{Introduction}

Lung cancer is one of the most common malignant tumors, exhibiting the highest mortality worldwide due to the uncontrolled cell growth in tissues of the lung and high metastatic ability (1). Non-small cell lung cancer (NSCLC) accounts for $80-85 \%$ of lung cancer (2). EGF receptor tyrosine kinase inhibitors (EGFR-TKI), such as gefitinib, have demonstrated notable therapeutic efficacy in non-small cell lung cancer patients $(3,4)$. However, nearly all patients develop drug resistance after a period of EGFR-TKI treatment, which eventually limits the application of EGFR-TKI (5). Therefore, developing strategies to overcome drug resistance is significant for improving prognosis and survival in the future.

A variety of molecular mechanisms are involved in the acquisition of TKIresistance,includingepithelial-mesenchymal transition (EMT) (6). EMT is a cellular process characterized by morphologic changes from an epithelial phenotype to a mesenchymal phenotype, by which cells lose epithelial cell-cell adhesions and gain the ability to move through the extracellular matrix. EMT is often associated with enhanced proliferation, invasion and metastasis in cancer cells (7). During EMT, cells exhibit the acquisition of mesenchymal markers, including Vimentin, type I collagen, fibronectin and Snail, and the inhibition of cell adhesion molecules, such as E-cadherin and catenin (8). Additionally, EMT is correlated with chemotherapeutic sensitivity in several types of cancer and sensitivity to EGFR-TKI in lung cancer cells, in particular (9-11). 
Napsin A, a member of the aspartic proteinase family, is frequently expressed in normal kidney and lung tissue, and is also present in lung adenocarcinoma (12-15). Napsin A was reported to be negatively associated with the malignancy degree of cancer cells (16-18). Cells with low Napsin A expression or without Napsin A expression appear to be prone to EMT (19). Hence, it can be speculated that EMT might be suppressed by Napsin A expression.

In the present study, Napsin A was overexpressed into the lung cancer A549 cell line its effect was investigated on their sensitivity to the EGFR-TKI, gefitinib. The results demonstrated that Napsin A expression combined with gefitinib has a synergistic inhibitory effect on cellular proliferation and a promotive effect on cell apoptosis of gefitinib-resistant A549s. In addition, Napsin A expression blocked the downregulation of E-cadherin and the upregulation of Vimentin expression in gefitinib-resistant cells. These data suggested that Napsin A resensitized the resistant A549 cells to gefitinib by reversing EMT and by inhibiting the activation of the integrin signaling pathway via focal adhesion kinase (FAK).

\section{Metarials and methods}

Cell culture. The human NSCLC cell line A549 was purchased from the Cell Bank of Type Culture Collection of the Chinese Academy of Sciences Institute (Shanghai, China). Cells were cultured in RPMI-1640 medium (Gibco; Thermo Fisher Scientific, Inc., Waltham, MA, USA) supplemented with $10 \%$ fetal bovine serum (FBS; Lonza Group, Ltd., Basel, Switzerland), $5 \mathrm{mM}$ L-glutamine, $5 \mathrm{mM}$ non-essential amino-acids, $100 \mathrm{U} / \mathrm{ml}$ penicillin and streptomycin (Invitrogen; Thermo Fisher Scientific, Inc.), in a humidified $5 \% \mathrm{CO}_{2}$ incubator at $37^{\circ} \mathrm{C}$. In preliminary experiments, A549 cells were detected to be sensitive to gefitinib and exhibited E-cadherin and vimentin expression, which facilitates the analysis of the EMT phenotype in response to Napsin A overexpression; whereas vimentin expression in H322, H358 and H441 lung cancer cells was negative or in trace amounts (data not shown). In addition, Napsin A expression levels in parental A549 cells are lower compared with other lung cancer cell lines (data not shown). Therefore, the A549 cell line was selected for the present study, with the hypothesis that exogenous overexpression of Napsin A in A549 cells might result in more significant change in biological function.

Development of the gefitinib-resistant A549 cell line. Gefitinib-resistant A549 cells (A549-GFT) were developed by exposing A549 cells to increasing concentration of gefitinib [(also known as Iressa and ZD1839; Bioruler, Beijing Bioway Biotechnology Co. Ltd., Beijing, China (http://a0002945. casmart.com.cn/)] ranging from 0.2 to $2 \mu \mathrm{mol} / 1$ in complete medium (20). Following exposure to increasing concentration of gefitinib for at least 3 months, live cells that developed resistance to $5 \mu \mathrm{mol} / 1$ gefitinib were collected, termed A549-GFT and used in subsequent experiments.

Morphological observation of gefitinib-resistant cells. Cells were cultured in $60 \mathrm{~mm}$ culture dishes for $24 \mathrm{~h}$, and then treated with or without $2 \mu \mathrm{mol} / 1$ gefitinib for $48 \mathrm{~h}$. The medium was then removed and cells were washed once with RPMI-1640 medium. Cell morphology was observed and photographed using a vertical microscope (Olympus Corporation, Tokyo, Japan).

Western blot analysis. Cells were treated with different doses of gefitinib as indicated. The cells were then washed twice with PBS and total protein was extracted using M-PER Mammalian Protein Extraction Reagent (Pierce; Thermo Fisher Scientific, Inc.) according to the manufacturer's manual. The proteins $(50 \mu \mathrm{g})$ were separated by $12 \%$ SDS-PAGE (Beijing Solarbio Science \& Technology Co., Ltd.) and transferred to polyvinylidene fluoride membranes (Millipore; Merck KGaA, Darmstadt, Germany). The membranes were blocked with $5 \%$ non-fat dried milk in TBST for $1 \mathrm{~h}$, and incubated with the following specific primary antibodies overnight at $4^{\circ} \mathrm{C}$ : Mouse monoclonal anti-E-cadherin (cat. no. sc-21791; 1:2,000), mouse monoclonal anti-Vimentin (cat. no. sc-6260; 1:2,000), anti-GAPDH (cat. no. sc-365062; 1:3,000; all Santa Cruz Biotechnology, Inc., Dallas, TX, USA), rabbit polyclonal anti-FAK (cat. no. 3285; 1:1,000), rabbit monoclonal anti-phospho-FAK (Tyr397; cat. no. 8556; 1:1,000; both Cell Signaling Technology, Inc., Danvers, MA, USA) and rabbit monoclonal anti-Napsin A (cat. no. ab133249; 1:10,000; Abcam, Cambridge, UK). Membrane were then incubated with horseradish peroxidase-conjugated secondary antibodies goat anti-mouse (cat. no. sc-2005; 1:2,000) and goat anti-rabbit IgG (cat. no. sc-2004; 1:2,000; both Santa Cruz Biotechnology, Inc.) for $2 \mathrm{~h}$ at room temperature. Development was performed using enhanced chemiluminescence detecting reagent (Amersham; GE Healthcare, Chicago, IL, USA). The protein blots were quantified by densitometry using QuantityOne software (Bio-Rad Laboratories, Inc., Hercules, CA, USA), and the amounts were expressed relative to the internal reference GAPDH.

Overexpression of Napsin A. The Napsin A-overexpressing plasmid, PLJM1-Napsin A, was constructed by cloning the full-length human Napsin A cDNA into the PLJM1 lentivirus vector (Addgene, Cambridge, CA, USA). cDNA was amplified using reverse transcription-polymerase chain reaction (RT-PCR). The PLJM1-Napsin A or the empty vehicle plasmid was cotransfected with pVSVG and pGag-pol plasmids (Clontech Laboratories, Inc., Mountainview, CA, USA) into HEK293T cells using Lipofectamine 2000 (Invitrogen; Thermo Fisher Scientific, Inc.) to produce virus, according to the manufacturer's manual. The lentivirus supernatant was harvested $48 \mathrm{~h}$ post-transfection and used to infect A549 cells in order to obtain Napsin A stable overexpressing cells. Puromycin (Sigma-Aldrich; Merck KGaA) was used to screen positive clones.

Proliferation assay. Cell proliferation was evaluated by 3-(4,5-dimethylthiazol-2-yl)-2,5-diphenyltetrazolium bromide (MTT; Sigma Aldrich; Merck KGaA) assay. A total of 2,000 cells were seeded into each well of a 96-well plate in $100 \mu 1$ of medium and incubated with or without various concentrations of gefitinib for different time periods at $37^{\circ} \mathrm{C}$ in a $5 \% \mathrm{CO}_{2}$ incubator. Then cells were incubated with $20 \mu \mathrm{l}$ of $5 \mathrm{mg} / \mathrm{ml}$ MTT for $4 \mathrm{~h}$, and then cells were lysed for $10 \mathrm{~min}$ by addition of $200 \mu \mathrm{l}$ dimethyl sulfoxide. Absorbance was measured at 
A

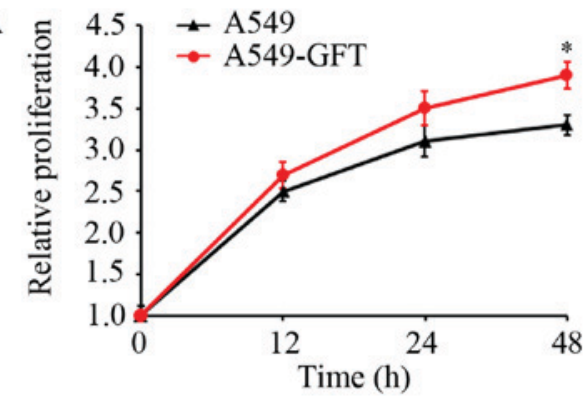

B

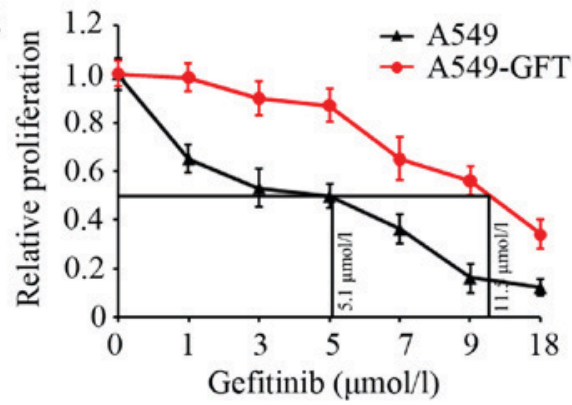

C

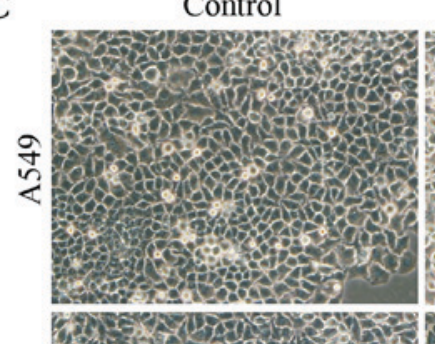

$2 \mu \mathrm{mol} / \mathrm{l}$ Gefitinib (48 h)

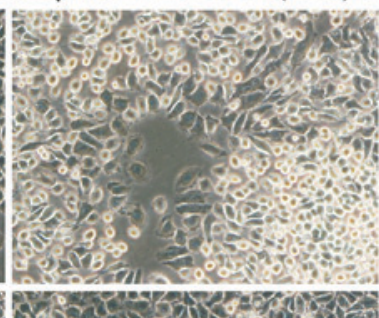

D A549 A549-GFT

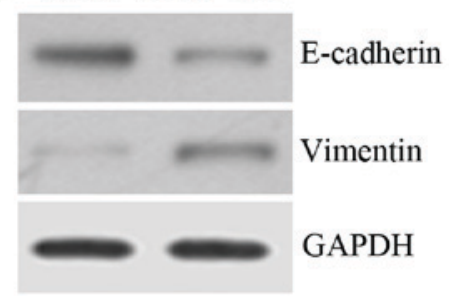

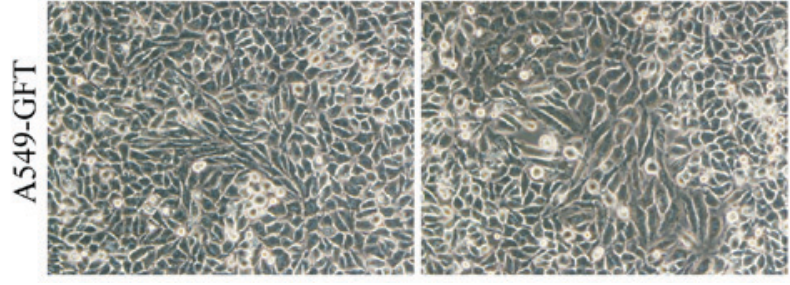

Figure 1. Characterization of A549-GFT and A549 cells. (A) Cells were maintained in 96-well plates and cell proliferation at indicated time intervals was assessed by MTT assay. Growth curves of the two cells were drawn. (B) Cells were incubated in $100 \mu 1$ medium for $24 \mathrm{~h}$, and then were treated with $0,1,3,5$, 7,9 and $18 \mu \mathrm{mol} / 1$ gefitinib for $48 \mathrm{~h}$. Cell proliferation was evaluated by MTT assay. The $\mathrm{IC}_{50}$ values of the two cell lines were calculated. (C) A549-GFT and A549 cells were exposed to $2 \mu \mathrm{mol} / 1$ gefitinib for $48 \mathrm{~h}$, and then cell morphology was visualized by microscopy. (D) E-cadherin and Vimentin expression in A549-GFT and A549 cells were assessed by western blot analysis. GAPDH was detected as an internal standard. "P<0.05 vs. A549 cells at 48 h.

490 nm using a Rainbow microplate reader (Tecan Group Ltd., Mannedorf, Switzerland). Cell proliferation was expressed as a $\%$ of the untreated control.

Apoptosis assay. Cells were grown to $80 \%$ confluence and treated with $5 \mu \mathrm{mol} / \mathrm{l}$ gefitinib for $48 \mathrm{~h}$. Apoptosis was analyzed using an Annexin V-fluorescein isothiocyanate (FITC)/propidium iodide (PI) assay (BD Biosciences, Franklin Lakes, NJ, USA) following the manufacturer's instructions. The amount of phosphatidylserine on the outer surface of the plasma membrane (a biochemical alteration unique to membranes of apoptotic cells) and the amount of PI (a dye that easily enters dead cells or cells in the late stages of apoptosis and binds DNA, but does not bind the plasma membrane of viable cells) were detected by flow cytometry using a FACSCalibur (BD Biosciences, San Jose, CA, USA). Data were analyzed using CellQuest software (BD Biosciences). Cells with phosphatidylserine on their surface were considered to be apoptotic.

Statistical analysis. All experiments were repeated at least in triplicate. Statistical analysis was performed with SPSS 11.0 (SPSS, Inc., Chicago, IL). Values are presented as the mean \pm standard error of the mean. One-way analysis of variance was used to assess differences between groups. The Duncan's new multiple range test was subsequently employed for pairwise comparisons followed by the Bonferroni correction. $\mathrm{P}<0.05$ was considered to indicate a statistically significant difference.

\section{Results}

Development of gefitinib-resistant A549 lung cancer cell line. To investigate the mechanism underlying gefitinib resistance in lung cancer, a gefitinib-resistant lung cancer cell model (A549-GFT) was first constructed by exposing the A549 lung cancer cell line to increasing concentrations of gefitinib. Cell growth was assessed using MTT assay and growth curves of A549-GFT and A549 cells were calculated. The data demonstrated that A549-GFT cells grew faster compared with A549 cells at the same time interval (Fig. 1A). Additionally, the $\mathrm{IC}_{50}$ of gefitinib was determined by exposing A549-GFT and A549 cells to different concentrations of gefitinib for $48 \mathrm{~h}$. The $\mathrm{IC}_{50}$ values of the two cells were respectively calculated to be 11.5 and $5.1 \mu \mathrm{mol} / 1$ (Fig. 1B). Further analysis of the gefitinib-resistant cells revealed that A549-GFT cells exhibited an EMT phenotype, characterized by spindle-like morphology, decreased E-cadherin expression and increased Vimentin expression (Fig. 1C and D). Finally, the two cell lines were treated with $2 \mu \mathrm{mol} / \mathrm{l}$ gefitinib for $48 \mathrm{~h}$ and their morphology was inspected by microscopy. As expected, A549 cells appeared round and with obvious blebbing, which are features of apoptosis (Fig. 1C). However, no significant changes in A549-GFT morphology were observed (Fig. 1C) 
following gefitinib treatment, confirming that the cells were gefitinib-resistant.

Napsin A overexpression resensitizes resistant A549 cells to gefitinib. To examine the effect of Napsin A on gefitinib resistance in lung cancer cells, the PLJM1-Napsin A expression vector was constructed and used to increase the expression of Napsin A. Western blot analysis confirmed that the Napsin A expression levels in the PLJM1-Napsin A-transduced A549 and A549-GFT cells were markedly elevated compared with the control cells (Fig. 2A). Next, A549 and A549-GFT cells were transiently transduced with empty control vector or PLJM1-Napsin A expression vector, and cultured in the presence or absence of $2 \mu \mathrm{mol} / 1$ gefitinib for $48 \mathrm{~h}$. Cell proliferation was then analyzed by MTT assay. The results demonstrated that cell proliferation of Napsin A-overexpressing A549-GFT cells was significantly suppressed by gefitinib treatment compared with gefitinib-treated control A549-GFT cells $(\mathrm{P}=0.006$; Fig. 2B). The growth of control A549-GFT cells was not nearly affected by gefitinib treatment and exhibited significantly increased survival compared with the parental A549 cells $(\mathrm{P}=0.019$; Fig. 2B). However, under identical experimental conditions, there was no significant difference in the proliferation between Napsin A-overexpressing and control A549 cells (Fig. 2B).

Napsin Aoverexpression promotes gefitinib-induced apoptosis in A549-GFT cells. To clarify the mechanism by which Napsin A overexpression resensitized the resistant A549-GFT cells to gefitinib, apoptosis was evaluated by the Annexin V-FITC/PI assay. A549-GFT and A549 cells were transduced with empty control and Napsin A-expressing vector, and exposed to $2 \mu \mathrm{mol} / 1$ gefitinib for $48 \mathrm{~h}$. The data indicated that gefitinib treatment induced the apoptosis of both Napsin A-overexpressing and control A549 cells compared to the corresponding untreated control cells $(\mathrm{P}=0.006$ and $\mathrm{P}=0.009$, respectively; Fig. $3 \mathrm{~A})$. Notably, Napsin A overexpression significantly stimulated the gefitinib-induced apoptosis in A549-GFT cells compared with the A549-GFT cells transduced with the empty vector $(\mathrm{P}=0.005$; Fig. 3A). However, gefitinib treatment did not significantly induce apoptosis in empty vector-transduced A549-GFT cells compared with empty vector-transduced parental A549 cells (Fig. 3A). These results suggest that Napsin A may be correlated with the resistance of lung cancer A549 cells to gefitinib and Napsin A expression may increase the sensitivity of resistant cells to gefitinib by enhancing gefitinib-induced apoptosis.

Additionally, the protein expression levels of E-cadherin, Vimentin and FAK were detected, because they serve a critical role in integrin-mediated signaling pathway and are correlated with cell transformation (21-23). Autophosphorylation of FAK at residue Tyr397 results in the activation of the integrin signaling pathway. The results demonstrated that Napsin A overexpression rescued the expression of E-cadherin and blocked the upregulation of Vimentin in A549-GFT cells (Fig. 3B). Furthermore, Napsin A overexpression resulted in an inhibition of FAK expression, as well as its phosphorylation at Tyr397 in resistant A549-GFT cells (Fig. 3B). These findings suggested that Napsin A reversed the EMT and enhanced the
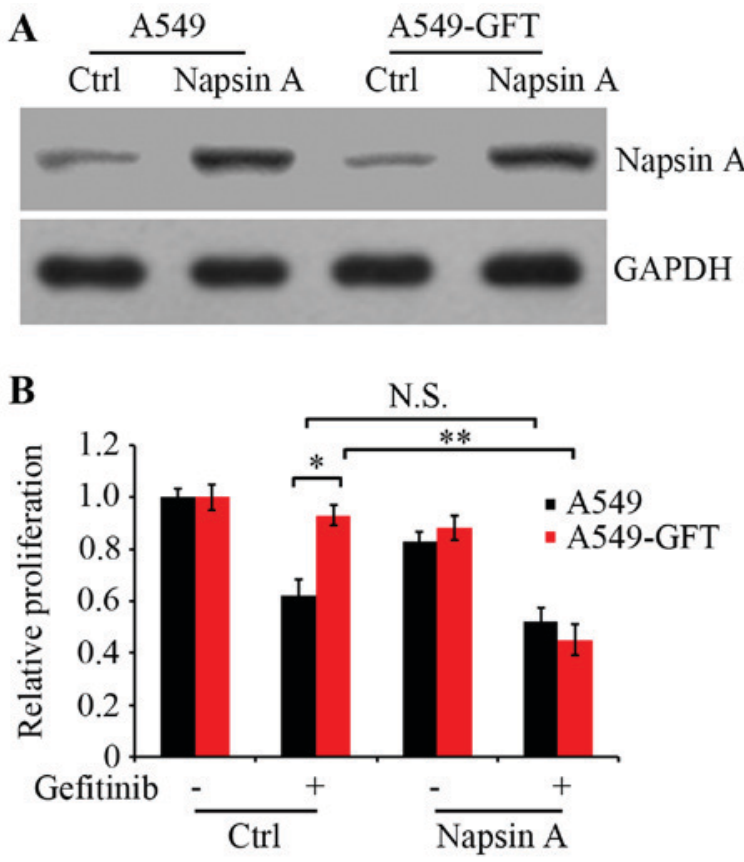

Figure 2. Effect of Napsin A overexpression on gefitinib sensitivity in A549-GFT cells. (A) Napsin A was overexpressed in A549-GFT and A549 cells by PLJM1-Napsin A lentivirus infection. Protein levels of Napsin A in Napsin A-overexpressing (Napsin A) and non-overexpressing control cells (Ctrl) were examined by western blot analysis. GAPDH was detected as an internal standard. (B) Cells were treated with or without $2 \mu \mathrm{mol} / 1$ gefitinib for 48 h. Cell proliferation was analyzed by MTT assay. ${ }^{*} \mathrm{P}<0.05$ and ${ }^{* *} \mathrm{P}<0.01$, with comparisons indicated by brackets. N.S., no significance.

sensitivity of A549-GFT cells to gefitinib, potentially through the inhibition of FAK-mediated integrin signaling.

\section{Discussion}

Lung cancer, a common malignancy with very high mortality, seriously threatens human health (1). The majority of lung cancers are diagnosed as NSCLC (2). EGFR-TKIs, such as gefitinib, have been used as the standard therapy in NSCLCs with EGFR-activating mutations $(3,4)$. However, nearly all patients eventually succumb to recurrence due to developing drug resistance; therefore the therapeutic efficacy of EGFR-TKIs is significantly limited. Therefore, exploring novel therapeutic strategies to overcome the acquisition of EGFR-TKI resistance is necessary. The present study demonstrated that Napsin A was able to attenuate the drug resistance of lung cancer A549 cells to gefitinib and Napsin A in combination with gefitinib significantly inhibited cancer cell growth in vitro.

In the present study, gefitinib-resistant lung cancer A549-GFT cells were firstly generated by simulating the condition of drug resistance development in vivo, using an increasing concentration of gefitinib over a long period of time. Results from a cell proliferation assay revealed that the $\mathrm{IC}_{50}$ of gefitinib in the drug-resistant A549-GFT cells at $48 \mathrm{~h}$ was markedly increased compared with the parental A549 cells. In addition, gefitinib treatment at $2 \mu \mathrm{mol} / 1$ concentration did not significantly affect the proliferation of A549-GFT cells, while under identical conditions, the proliferation of the parental A549 cells was reduced by $\sim 80 \%$. These findings confirmed that A549-GFT cells were resistant to gefitinib and 

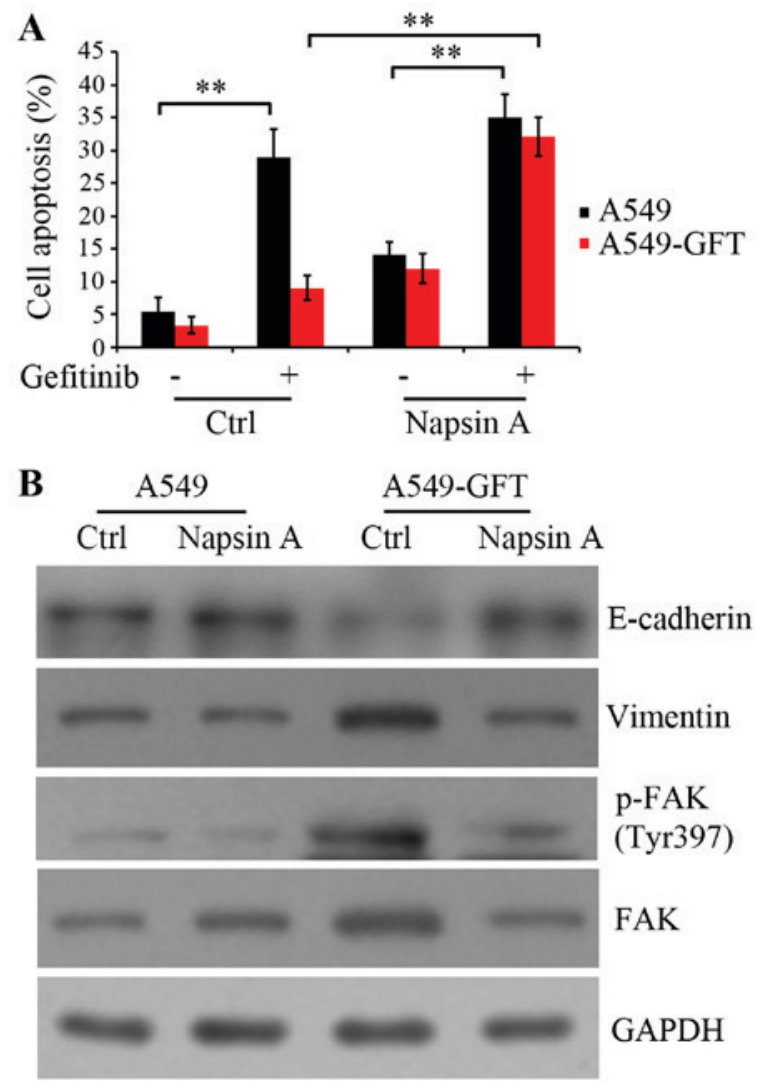

Figure 3. Effect of Napsin A overexpression on gefitinib-induced apoptosis in A549-GFT cells. Napsin A was overexpressed in A549 and A549-GFT cells by PLJM1-Napsin A lentivirus infection, and the cells were then exposed to $2 \mu \mathrm{mol} / 1$ gefitinib for $48 \mathrm{~h}$. (A) Apoptosis of A549 and A549-GFT cells was evaluated by Annexin V/propidium iodide staining assay and flow cytometry (B) Western blot analysis was used to detect the protein expression levels of E-cadherin, Vimentin, p-FAK and total FAK. GAPDH was detected as an internal standard. ${ }^{* *} \mathrm{P}<0.01$, with comparisons indicated by brackets. FAK, focal adhesion kinase; p-, phosphorylated; NS, no significance.

could be used for subsequent experiments. Notably, gefitinib resistance was demonstrated to cause changes in A549 cell morphology, from cobblestone-like epithelial cells into slender spindle-shaped cells, suggesting that the resistant cells had undergone. EMT is characterized by the loss of cell adhesion molecules, such as E-cadherin, and elevation of mesenchymal markers, such as Vimentin (8). The present results demonstrated that gefitinib-resistant A549-GFT cells displayed downregulation of E-cadherin expression and upregulation of Vimentin expression compared with parental A549 cells. These data supported that the development of resistance to gefitinib in A549 cells induced EMT. A previous study has indicated that EMT is involved in the acquisition of TKI resistance (6). Hence, it was speculated that EMT might be the mechanism underlying the acquisition of gefitinib resistance in A549 cells.

Napsin A has been reported to inhibit EMT in A549 cells (24). In the present study, Napsin A-overexpressing A549 and gefitinib-resistant A549-GFT cell lines were constructed. It was demonstrated that Napsin A overexpression remarkably suppressed EMT in gefitinib-resistant cells, resulting in the restoration of E-cadherin expression and the downregulation of Vimentin expression. Furthermore, Napsin A overexpression significantly enhanced the sensitivity of resistant A549-GFT cells to gefitinib by inhibiting cell proliferation and inducing cell apoptosis. In addition, the results demonstrated that FAK was inhibited following Napsin A overexpression in resistant A549-GFT cells. FAK has an important role in integrin signaling $(21,25-27)$. FAK gets activated by integrin and subsequently regulates several signaling pathways, including signal transducer and activator of transcription 1 (STAT1), mitogen-activated protein kinase (MAPK) and phosphoinositide 3-kinase (PI3K) signaling (21-23), resulting in the initiation of cell proliferation and transformation. Integrin aggregation by ligand binding results in the oligomerization of FAK, which is mediated by talin. Autophosphorylation of FAK at residue Tyr397 results in the binding of the SH2 domain of Src and Fyn, which then phosphorylate a number of FAK-associated proteins, including paxilin, tensin and the docking protein p130CAS (Crk-Associated Substrate). In addition, phosphorylation of Tyr397 leads to the recruitment of other SH2-containing proteins, including $\mathrm{PI} 3 \mathrm{~K}$, phospholipase- $\mathrm{C} \gamma$ and the adapter protein growth factor receptor-bound protein 7 (GRB7) (5), this resulting in the activation of the integrin signaling pathway (28). The carboxyl terminal of the Napsin A contains an Arg-Gly-Asp (RGD) sequence, which recognizes and binds integrins on the cell surface (29). Integrins mediate cell adhesion and signal transduction between cells and the extracellular matrix (ECM) (30), and they are an important regulator of cell proliferation, apoptosis, migration, and metastasis $(31,32)$. In the present study, it may be possible that Napsin A repressed the interaction between integrins and ECM through RGD sequence-mediated interaction with integrin, thus hindering integrin signaling pathway and inhibiting cell proliferation and transformation by downregulating FAK expression and phosphorylation in gefitinib-resistant A549-GFT cells. Further investigation needs to be performed in order to test this hypothesis.

In conclusion, the present data demonstrated that Napsin A overexpression combined with the EGFR-TKI gefitinib may be an effective method to improve the sensitivity of lung cancer cells to gefitinib. Napsin A could be delivered to tumor tissues through viral vectors or liposomes in order to elevate its expression level as a potential clinical gene treatment strategy for patients with gefitinib-resistant lung tumors. Future studies will employ additional gefitinib-resistant lung cancer cell lines in order to confirm that this effect is not specific to A549 cells, and to further investigate the association between gefitinib resistance and Napsin A expression in lung cancer.

\section{Acknowledgements}

Not applicable.

\section{Funding}

No funding was received.

\section{Availability of data and materials}

The datasets used and/or analyzed during the current study are available from the corresponding author on reasonable request. 


\section{Authors' contributions}

$\mathrm{LZ}, \mathrm{XL}$ and ZW were major contributors in the conception and design of the research and revision of the manuscript for important intellectual content. Acquisition of data was performed by JY. YZ and TX were the major contributors in the analysis and interpretation of data and statistical analysis. Drafting the manuscript was performed by ZW.

\section{Ethics approval and consent to participate}

Not applicable.

\section{Consent for publication}

Not applicable.

\section{Competing interests}

The authors declare that they have no competing interests.

\section{References}

1. PDQ Adult Treatment Editorial Board: Non-small cell lung cancer treatment $(P D Q \circledR)$ : Patient Version. PDQ cancer information summaries [Internet]. Nat Cancer Inst (US), Bethesda. https://www.ncbi.nlm.nih.gov/books/NBK65917/ Accessed April 13, 2017.

2. Pao W and Girard N: New driver mutations in non-small-cell lung cancer. Lancet Oncol 12: 175-180, 2011.

3. Soria JC, Mok TS, Cappuzzo F and Jänne PA: EGFR-mutated oncogene-addicted non-small cell lung cancer: Current trends and future prospects. Cancer Treat Rev 38: 416-430, 2012.

4. Nguyen KS and Neal JW: First-line treatment of EGFR-mutant non-small-cell lung cancer: The role of erlotinib and other tyrosine kinase inhibitors. Biologics 6: 337-345, 2012.

5. Pao W, Miller VA, Politi KA, Riely GJ, Somwar R, Zakowski MF, Kris MG and Varmus H: Acquired resistance of lung adenocarcinomas to gefitinib or erlotinib is associated with a second mutation in the EGFR kinase domain. PLoS Med 2: e73, 2005.

6. Wu PF, Zhu YP, Yang CH, Wang YF and Wang GH: The Mechanism and countermeasures on the secondary resistance of Epidermal Growth Factor Receptor Tyrosine Kinase Inhibitor (EGFR-TKI). Anti-Tumor Pharm 5: 4, 2015.

7. Thiery JP, Acloque H, Huang RY and Nieto MA: Epithelial-mesenchymal transitions in development and disease. Cell 139: 871-890, 2009.

8. Robert G, Gaggioli C, Bailet O, Chavey C, Abbe P, Aberdam E, Sabatié E, Cano A, Garcia de Herreros A, Ballotti R and Tartare-Deckert S: SPARC represses E-cadherin and induces mesenchymal transition during melanoma development. Cancer Res 66: 7516-7523, 2006

9. Voulgari A and Pintzas A: Epithelial-mesenchymal transition in cancer metastasis: Mechanisms, markers and strategies to overcome drug resistance in the clinic. Biochim Biophys Acta 1796 75-90, 2009 .

10. Neel DS and Bivona TG: Secrets of drug resistance in NSCLC exposed by new molecular definition of EMT. Clin Cancer Res 19: 3-5, 2013.

11. Uramoto $H$, Iwata $T$, Onitsuka $T$, Shimokawa $H$, Hanagiri $T$ and Oyama T: Epithelial-mesenchymal transition in EGFR-TKI acquired resistant lung adenocarcinoma. Anticancer Res 30: 2513-2517, 2010

12. Tatnell PJ, Powell DJ, Hill J, Smith TS, Tew DG and Kay J: Napsins: New human aspartic proteinases. Distinction between two closely related genes. FEBS Lett 441: 43-48, 1998.
13. Chuman Y, Bergman A, Ueno T, Saito S, Sakaguchi K, Alaiya AA, Franzén B, Bergman T, Arnott D, Auer G, et al: Napsin A, a member of the aspartic protease family, is abundantly expressed in normal lung and kidney tissue and is expressed in lung adenocarcinomas. FEBS Lett 462: 129-134, 1999.

14. Schauer-Vukasinovic V, Bur D, Kling D, Grüninger F and Giller T: Human napsin A: Expression, immunochemical detection, and tissue localization. FEBS Lett 462: 135-139, 1999.

15. Hirano T, Auer G, Maeda M, Hagiwara Y, Okada S, Ohira T, Okuzawa K, Fujioka K, Franzén B, Hibi N, et al: Human tissue distribution of TA02, which is homologous with a new type of aspartic proteinase, napsin A. Jpn J Cancer Res 91: 1015-1021, 2000.

16. Hirano T, Gong Y, Yoshida K, Kato Y, Yashima K, Maeda M, Nakagawa A, Fujioka K, Ohira T, Ikeda N, et al: Usefulness of TA02 (napsin A) to distinguish primary lung adenocarcinoma from metastatic lung adenocarcinoma. Lung Cancer 41: 155-162, 2003.

17. Ueno T, Linder S and Elmberger G: Aspartic proteinase napsin is a useful marker for diagnosis of primary lung adenocarcinoma. Br J Cancer 88: 1229-1233, 2003.

18. Hirano T, Auer G, Maeda M, Hagiwara Y, Okada S, Ohira T, Okuzawa K, Fujioka K, Franzén B, Hibi N, et al: Human tissue distribution of TA02, which is homologous with a new type of aspartic proteinase, napsin A. Jpn J Cancer Res 91: 1015-1021, 2000.

19. Ueno T,Elmberger G, WeaverTE, Toi M and Linder S: The aspartic protease napsin A suppresses tumor growth independent of its catalytic activity. Lab Invest 88: 256-263, 2008.

20. Meena AS, Sharma A, Kumari R, Mohammad N, Singh SV and Bhat MK: Inherent and acquired resistance to paclitaxel in hepatocellular carcinoma: Molecular events involved. PLoS One 8: e61524, 2013

21. Hauck CR, Hsia DA and Schlaepfer DD: The focal adhesion kinase-a regulator of cell migration and invasion. IUBMB Life 53: 115-119, 2002.

22. Xie B, Zhao J, Kitagawa M, Durbin J, Madri JA, Guan JL and Fu XY: Focal adhesion kinase activates Stat1 in integrin-mediated cell migration and adhesion. J Biol Chem 276: 19512-19523, 2001.

23. Bhowmick NA, Zent R, Ghiassi M, McDonnell M and Moses HL: Integrin beta 1 signaling is necessary for transforming growth factor-beta activation of p38MAPK and epithelial plasticity. J Biol Chem 276: 46707-46713, 2001.

24. Zheng JX, Guan SH, Xu Q, Liu JZ and Song P: Inhibition of epithelial-mesenchymal transition in A549 cell by transfected Napsin A. Chin Med J (Engl) 125: 2734-2740, 2012.

25. Hauck CR, Sieg DJ, Hsia DA, Loftus JC, Gaarde WA, Monia BP and Schlaepfer DD: Inhibition of focal adhesion kinase expression or activity disrupts epidermal growth factor-stimulated signaling promoting the migration of invasive human carcinoma cells. Cancer Res 61: 7079-7090, 2001.

26. Sieg DJ, Hauck CR, Ilic D, Klingbeil CK, Schaefer E, Damsky CH and Schlaepfer DD: FAK integrates growth-factor and integrin signals to promote cell migration. Nat Cell Biol 2: 249-256, 2000

27. Sieg DJ, Hauck CR and Schlaepfer DD: Required role of focal adhesion kinase (FAK) for integrin-stimulated cell migration. J Cell Sci 112: 2677-2691, 1999.

28. Parsons JT, Martin KH, Slack JK, Taylor JM and Weed SA: Focal adhesion kinase: A regulator of focal adhesion dynamics and cell movement. Oncogene 19: 5606-5613, 2000.

29. Ruoslahti E: RGD and other recognition sequences for integrins. Annu Rev Cell Dev Biol 12: 697-715, 1996.

30. Juliano RL: Signal transduction by cell adhesion receptors and the cytoskeleton: Functions of integrins, cadherins, selectins, and immunoglobulin-superfamily members. Annu Rev Pharmacol Toxicol 42: 283-323, 2002.

31. Hynes RO: Integrins: Bidirectional, allosteric signaling machines. Cell 110: 673-687, 2002.

32. Li Y, Yang J, Dai C, Wu C and Liu Y: Role for integrin-linked kinase in mediating tubular epithelial to mesenchymal transition and renal interstitial fibrogenesis. J Clin Invest 112: 503-516, 2003. 